\title{
The Bidirectional Relationship
} between Positive Mental Health and Social Rhythm in College Students: A Three-Year Longitudinal Study

\author{
Dan Cai ${ }^{1}$, Meixia Zhu' ${ }^{1}$, Muyu Lin ${ }^{2 *}$, Xiao Chi Zhang ${ }^{2}$ and Jürgen Margraf ${ }^{2}$ \\ ${ }^{1}$ Department of Psychology, Shanghai Normal University, Shanghai, China, ${ }^{2}$ Department of Clinical Psychology and \\ Psychotherapy, Mental Health Research and Treatment Center, Ruhr-Universität Bochum, Bochum, Germany
}

Social rhythm refers to the general regularity of engaging in basic social activities during the week, and was found to be associated with individuals' positive mental health. The present study investigated the relationship between social rhythm and emotional well-being in a cohort of 2,031 college students over 3 years with a cross-lagged longitudinal panel design. Results revealed that regularity of social rhythm positively predicted emotional well-being in the following year, and vice versa, when the level of both factors in the previous year was controlled. Our study provides evidence of

OPEN ACCESS

Edited by:

Gian Mauro Manzoni, Università degli Studi eCampus, Italy

Reviewed by:

Deana Davalos,

Colorado State University, United States

Silvia Serino, Università Cattolica del Sacro Cuore,

${ }^{*}$ Correspondence: Muyu Lin

muyud@pku.edu.cn

Specialty section:

This article was submitted to Clinical and Health Psychology, a section of the journal Frontiers in Psychology

Received: 23 March 2017 Accepted: 16 June 2017

Published: 30 June 2017

Citation:

Cai D, Zhu M, Lin M, Zhang XC and Margraf J (2017) The Bidirectional Relationship between Positive Mental Health and Social Rhythm in College

Students: A Three-Year Longitudinal Study. Front. Psychol. 8:1119. doi: 10.3389/fpsyg.2017.01119 a longitudinal positive reciprocal relationship between social rhythm and positive mental health in younger adult populations.

Keywords: social rhythm, positive mental health, cross-lagged panel analysis, college students, longitudinal study

\section{INTRODUCTION}

It is well-known that physical health and psychological health are closely linked with each other. For instance, regular biological daily patterns, such as temperature fluctuations and the circadian rhythm play a key role in maintaining good mental health, whereas disruption or irregularity in time-cues that trigger one's biological and social behavior, such as changes in the duration of daylight, sleep/wake cycles, social contacts, working schedules, and environment signals, can lead to increasing symptoms of mental illnesses (Ehlers et al., 1988; Costa, 1996; Thase et al., 2002; Grandin et al., 2006; Gorwood, 2012; Lieverse et al., 2013).

Among all physical and social factors related to mental health, the influence of social rhythm patterns - rhythmic social and behavioral patterns in daily routines that may be directly or indirectly related to other people such as mealtimes, bedtimes, and social interaction during weekdays and weekends - might be underestimated, but in fact they have repeatedly been shown to be related to individuals' mental health (Ehlers et al., 1988; Sylvia et al., 2009). Previous studies regarding social rhythm mainly focused on its relationship with psychopathology, especially with depression (Brown et al., 1996; Lieverse et al., 2013) and bipolar disorder (Grandin et al., 2006; Meyer and Maier, 2006; Bullock et al., 2011). For instance, individuals with bipolar spectrum disorders experienced greater disruption of their social rhythm than controls following both positive and negative life events (e.g., the birth of an infant or losing a spouse or job) (Boland et al., 2012). Further, less social rhythm regularity was found to predict more rapid reoccurrence of affective episodes (i.e., less time in a euthymic state) in bipolar individuals during the prospective follow-up (Shen et al., 2008). And, as proposed in the Social Zeitgeber Theory (Ehlers et al., 1988), disruption of one's time cues that prompt human circadian rhythms changes the stability 
of social and biological rhythms, resulting in more symptoms or episodes of mental illness. On the other hand, promoting life rhythm in therapy was also proven effective in treating bipolar disorder, marked by fast stabilization, longer episode-free periods, and decreased disorder recurrence (Frank et al., 2005; Nusslock and Frank, 2012). Beyond affective disorders, there are indications of a relationship between social rhythm irregularity and posttraumatic stress disorder (PTSD). Similarly, many patients with PTSD experience disturbances in their sleep pattern (Harvey et al., 2003; Kobayashi et al., 2007; Haynes et al., 2016), and psychotherapy aiming to enhance social rhythm regularity has demonstrated promising results in reducing symptoms and increasing sleep quality in these patients (Haynes et al., 2016).

As reviewed above, a solid body of research has shed light on the understanding of the bidirectional relationship between social rhythm and mental illness, especially affective diseases. According to the World Health Organization (World Health Organization, 2004), however, good mental health is not only the absence of psychopathology, but additionally " a state of wellbeing in which the individual realizes his or her own abilities, can cope with the normal stresses of life, can work productively and fruitfully, and is able to make a contribution to his or her community" (p.12). In the recent decades, the positive aspects of mental health are taking a more prominent role in public mental health care and disorder-related research and practice. Accumulating studies have shown that the negative and positive aspects of mental health are only moderately related to each other, and that the association between the two is bidirectional, supported by the two-dimensional model of mental health (Westerhof and Keyes, 2010; Kendler et al., 2011; Weich et al., 2011; Lamers et al., 2015). Furthermore, positive mental health emerged as a predictor of the remitting course of mental disorders (Lukat et al., 2017) and the remission of suicidal thoughts in young women after 17 months (Teismann et al., 2016).

To our knowledge, only a few studies have investigated the relationship between social rhythm and positive mental health. For instance, a cross-sectional study with a representative German sample of over 7,000 people revealed that social rhythm irregularity was associated with less life satisfaction and greater depression, anxiety, and stress (Velten et al., 2014). A recent large-scale research study with samples from Russia, Germany, and the United States demonstrated that social rhythm regularity was an important cross-cultural factor in predicting higher positive mental and physical health, as well as less health problems (Margraf et al., 2016). Similarly, a relatively regular working schedule is positively linked to psycho-social well-being, such as family and social commitments. Moreover, individuals who engaged in regular physical activity displayed not only decreased mood symptoms but also enhanced experience of wellbeing (Penedo and Dahn, 2005). Sleep hygiene, which involved regular sleep schedules, was found to partially or indirectly predict depression and poor well-being in college students (Peach et al., 2016).

Most previous studies focusing on social rhythm and positive mental health adopted a cross-sectional design (Velten et al., 2014; Margraf et al., 2016), leaving the question unresolved whether the strong association found between these two constructs was due to the fact that a regular social rhythm would lead to a higher level of positive mental health, or vice versa. Logically, the next step should be to investigate the longitudinal and potential causal relationship between the two important constructs (Kraemer et al., 1997). The few longitudinal studies so far mainly focused on either the predictor role social rhythm plays on negative mental health (Frank et al., 2005; Shen et al., 2008; Nusslock and Frank, 2012), or, in the opposite direction, how mental illness symptoms predict social rhythm (Boland et al., 2012), suggesting a bidirectional relationship between social rhythm and psychopathological symptoms. Meanwhile, it remains unknown whether social rhythm regularity and positive mental health would also benefit from each other over time.

Therefore, the present study aimed to gain insight into the reciprocal relationship of social rhythm regularity predicting higher levels of positive mental health, and vice versa. The Brief Social Rhythm Scale (BSRS) (Margraf et al., 2016) and Positive Mental Health Scale (PMHS, Lukat et al., 2016) were applied in a large college student sample in China over three, sequential 12 -month periods. The BSRS is a new tool with multiple language versions that quickly assesses rhythmicity in eating, sleeping, and socializing patterns. The PMHS was applied, as a valid short unidimensional measure of general emotional well-being (Lukat et al., 2016). A large-scale population diary study revealed that the highest rhythmicity was found within older people and those who cohabite with a partner and children, while younger single populations were relatively less rhythmic (van Tienoven et al., 2014). Given that the academic pressures and life changes of college life may also lead to greater risk of irregularity in social rhythm as well as to changes in emotional well-being, a college student sample was selected for the current study. Furthermore, the survey continued for 3 years in order to track changes in both social rhythm and positive mental health over time.

Based on earlier empirical evidence that social rhythm and positive mental health were associated with each other crosssectionally (Margraf et al., 2016) and that social rhythm shared a possible reciprocal relationship with mental illness (Frank et al., 2005; Shen et al., 2008; Boland et al., 2012; Nusslock and Frank, 2012), we hypothesized that (1) baseline (T1) social rhythm regularity would positively correlate with baseline positive mental health score; (2) scores on BSRS at T1 would predict scores on BSRS at 1-year-follow-up (T2), and T2 BSRS scores would predict 2-year-follow-up (T3) BSRS scores; (3) PMHS at T1 would also predict PMHS at T2, and PMHS at T2 would predict PMHS at T3; (4) baseline social rhythm would predict change in PMHS at T2, as well as baseline PMHS would predict social rhythm at T2; and (5) change in social rhythm at T2 would predict change in PMHS at T3, and vice versa that PMHS at T2 would predict social rhythm at $\mathrm{T} 3$.

\section{MATERIALS AND METHODS}

\section{Participants}

This project was a part of Bochum Optimism and Mental Health (BOOM) research project (Velten et al., 2014; Margraf 
et al., 2016). All participants were students at Shanghai Normal University, China. This university offers 17 major programs, the current project randomly selected students from five of the programs, including majors from humanities, sciences, etc. The project lasted for three academic years (from 2013 to 2015) with one distribution of questionnaires each year (altogether three waves of surveys, referred as T1, T2, and T3, respectively). Each survey was conducted in May, which was around the middle of the second semester of the academic year, in order to avoid potential influences of the beginning or end of a new academic year or new semester on the psychological or social well-being of participants. The sample size for T1 was 2,985; 2,992 for T2, and 2,818 for T3. Participants who completed less than $80 \%$ of the two target scales, who were suspected not to respond sincerely (i.e., all the answers were the same; e.g., chose 1 in all 9 items of PMHS), or who missed one or more surveys were excluded. Finally, answers from 2,031 participants $(68.04 \%$ of the initial sample) were included for further statistical analyses. Missing values were replaced by mean values. Among them, 1,621 were females, and 410 were males. At baseline, 1,177 students were in their freshman year, 851 were sophomore year students, and 3 were in their junior year of college. The average age (at T1) of the longitudinal sample was $19.89 \pm 0.91$, ranging from 17 to 29 .

\section{Measures}

\section{The Brief Social Rhythm Scale (BSRS)}

The BSRS assesses the regularity of participants' 10 basic daily activities, such as mealtimes, bedtimes, and walking times during the work week/school week and on the weekend (Margraf et al., 2016). General regularity of each activity is rated with a 6-point scale ranging from 1 (very regularly) to 6 (very irregularly). Items of the BSRS can be found in Appendix A. Final scores are recoded reversely, therefore higher scores of BSRS indicate higher regularity in social rhythm. The internal consistency was 0.80 , 0.86 , and 0.89 , for $\mathrm{T} 1, \mathrm{~T} 2$, and $\mathrm{T} 3$ based on the responds of the longitudinal sample, respectively.

\section{Positive Mental Health Scale (PMHS)}

The PMHS is a 9-item scale measuring a holistic concept of emotional well-being related to positive mental health (Lukat et al., 2016). Participants respond on a 4-point Likert-type scale, ranging from 0 (I disagree) to 3 (I agree). Items of the PMHS can be found in Appendix B. This scale has been applied in several different samples (students and different patient samples), showing strong psychometric properties and a one-dimensional structure. In a major validation study on student, patients, and general samples, PMHS showed a 1-week retest reliability of 0.81 (general adults sample) and an internal consistency of 0.93 (student sample) (Lukat et al., 2016). The Cronbach's $\alpha$ of the longitudinal sample was 0.88 (T1), 0.90 (T2), and 0.94 (T3).

\section{Data Collection}

The study was approved by the Ethics Committee of the Faculty of Psychology of the Ruhr-Universität Bochum and the Academic Ethics Committee of the Shanghai Normal University. Study aims, anonymity, voluntariness, and other information of the survey were informed in the advertisement of the study spread across the college campus. Students who agreed with the terms would voluntarily come to the computer rooms of the university within a certain time range specified in the advertisement to participate the study. When they entered the computer rooms, the aims of the study, voluntariness, and anonymity information were explained again by survey conductors, who were two trained graduate students majoring in psychology. Participants gave oral consent one by one, those who did not give consent left the computer room directly. Then, the survey conductors opened the webpage of the online questionnaire for participants to fill in. The online questionnaire did not ask for information related to participants personal ID. Therefore, no other written consent forms were exchanged. This consent procedure was approved by the Academic Ethics Committee of the Shanghai Normal University.

Participants completed the survey in different waves (50-80 per wave) during the same day. Demographic information questionnaire, PMHS, BSRS, and other questionnaires from the BOOM project were used for each survey. Completing the entire questionnaire battery took approximately $30 \mathrm{~min}$. Participants gave their consent before participation and received a gift (about \$ 1.5) after completing each survey.

\section{Statistical Analysis}

Correlations between social rhythm and positive mental health (at T1, T2, and T3) were computed as a primary test for the relationship between the two target variables. Then, the mean changes in social rhythm and positive mental health across the 3 years were tested via repeated-measures multivariate analysis of variance (MANOVA) using SPSS (version 19.0; IBM Corp, 2010).

Scores on BSRS and PMHS at T1, T2, and T3 were included for modeling and examining intra-individual changes over time using a cross-lagged panel analysis. The panel analysis was performed using Amos22.0 (Arbuckle, 2013). Cross-lagged path coefficients (i.e., predictive associations) between T1 and T2 for social rhythm (measured by BSRS) and positive mental health (indexed by PMHS) were included as well as the path coefficients between $\mathrm{T} 2$ and $\mathrm{T} 3$. The panel model further included stability coefficients between T1, T2, and T3 for SBRS and PMHS in order to allow follow-up measurements (T2 and T3) to reflect residual change over time. We further included correlations (non-direction al associations) between social rhythm and positive mental health at each of the three time points (for T2 and T3, correlations were between residual errors) and the correlation between social rhythm at $\mathrm{T} 1$ and at T3 as well as the correlation between positive mental health at T1 and at T3 (Model 1). Overall model fit was assessed using chi square test, comparative fit index (CFI), and standardized root mean square residual (SRMR). CFI $\geq 0.95$ and $\mathrm{SRMR} \leq 0.08$ together indicate good model fit ( $\mathrm{Hu}$ and Bentler, 1999). Alpha level of significance of all path coefficients and correlations was set at 0.05 .

In order to examine the potential influence of gender, another two multi-group cross-lagged panel analyses were conducted and 
compared. In Model 2, the structures of both gender groups were identical as in Model 1, and all the path coefficients were freely estimated. Then in Model 3, the structures were identical with Model 2, but all the respective path coefficients were constrained to be equal across both gender groups. $\chi^{2}$ differences were computed to determine whether Model 2 and Model 3 differed from each other.

\section{RESULTS}

\section{Relations between Positive Mental Health and Social Rhythm}

The means and standard deviations (SDs) of the BSRS and the PMHS scores at the three time points are described in Table $\mathbf{1 .}$ Bivariate correlations among the scores of PMHS and BSRS in 3 years are shown in Table 1, indicating that scores from the PMHS and BSRS were significantly correlated per time point (i.e., at T1, $r=0.33$ ) and across time points (e.g., for T1 PMHS and T2 BSRS, $r=0.22$ ).

\section{Changes in Positive Mental Health and Social Rhythm across Time}

A repeated-measures MANOVA was conducted with the time (T1, T2, and T3) as within group independent variable and the scores on the BSRS and scores on the PMHS as dependent variables. The Mauchly's test of sphericity was violated, therefore Huynh-Feldt epsilon correction was applied. Bonferroni correction was used for pairwise comparisons.

For PMHS, a significant effect of time was observed, $F(1.97,3991.03)=96.29, p<0.0001, \eta_{p}^{2}=0.05$. Pairwise comparisons showed that PMHS scores at T1 was higher than the scores at T2, Mean $n_{\text {difference }}=1.38, S E_{\text {difference }}=0.09, p<0.0001$, $d=0.32$, and at T3, Mean $n_{\text {difference }}=0.84, S E_{\text {difference }}=0.11$, $p<0.0001, d=0.19$; and that the scores of PMHS at T2 was lower than that at T3, Mean $n_{\text {difference }}=-0.55, S E_{\text {difference }}=0.10$, $p<0.00001, d=0.12$.

For BSRS, a significant effect of time was found, $F(1.97,3998.08)=43.05, p<0.0001, \eta_{\mathrm{p}}^{2}=0.02$. Further analyses indicated that the social rhythm regularity decreased significantly year by year: the social rhythm regularity at $\mathrm{T} 1$ was more regular than that at T2 $\left(\right.$ Mean $_{\text {difference }}=0.55, S E_{\text {difference }}=0.17$, $p=0.003, d=0.07)$ and at T3 $\left(\right.$ Mean $_{\text {difference }}=1.62$, $S E_{\text {difference }}=0.18, p<0.0001, d=0.22$ ). In addition, the social rhythm measured at T2 was also more regular than measured at T3, Mean $n_{\text {difference }}=1.08, S E_{\text {difference }}=0.18$, $p<0.0001, d=0.14$.

\section{Cross-Lagged Panel Modeling}

The PMHS and BSRS were simultaneously examined in a parallel process using cross-lagged panel Model 1 (Figure 1). The model was a good fit to the data, $\chi^{2}(2)=34.01, p<0.0001, \mathrm{CFI}=0.99$, and SRMR $=0.029$. The standardized path coefficients of the model are provided in Figure 1. As expected for BSRS, the autoregressive regression estimates were positive, strong, and significant from $\mathrm{T} 1$ to $\mathrm{T} 2$ and from $\mathrm{T} 2$ to $\mathrm{T} 3$. The same was found for PMHS from T1 to T2 and from T2 to T3, indexing also by positive, strong, and significant autoregressive regression estimates. The cross-lagged parameter estimates of social rhythm at T1 and T2 to PMHS at T2 and T3, respectively, were positive and significant at 0.01 and 0.001 level, suggesting that social rhythm regularity could predict positive mental health even when controlling for the scores from the previous years. Meanwhile, the path coefficients from PMHS at T1 and T2 to BSRS at T2 and $\mathrm{T} 3$, respectively, were also positive and reached a 0.001 level of significance, indicating that positive mental health was also a strong predictor of social rhythm in college students.

\section{Potential Gender Effect}

After dividing the sample into female and male subgroups, Model 2 and Model 3 were conducted based on both gender groups. Model fit comparison results showed that both models did not significantly differ from each other, $\Delta \chi^{2}=8.42, d f=8, p=0.39$. This result indicated that the path coefficient values in both gender groups were similar, thus the results of Model 1 were representative for both genders.

\section{DISCUSSION}

The present study was the first study to examine the bidirectional relationship between social rhythm and positive mental health over 3 years using cross-lagged panel analysis in a college student sample. The large sample size $(N=2031)$ in our study provided high power to detect even small effects. Results showed that social rhythm regularity decreased year by year, while emotional well-being seemed to fluctuate from decreasing at T2 and then increasing again at T3. As expected, our analyses further revealed a significant reciprocal relationship between social rhythm and

TABLE 1 | Means and standard deviations (SDs) descriptions and correlations between positive mental health and social rhythm at each time point.

\begin{tabular}{|c|c|c|c|c|c|c|c|}
\hline & Mean & $S D$ & PMHS (T1) & PMHS (T2) & PMHS (T3) & BSRS (T1) & BSRS (T2) \\
\hline PMHS (T1) & 21.91 & 4.19 & & & & & \\
\hline PMHS (T2) & 20.53 & 4.38 & $0.52^{* *}$ & & & & \\
\hline PMHS (T3) & 21.07 & 4.64 & $0.43^{* *}$ & $0.48^{* *}$ & & & \\
\hline BSRS (T1) & 46.87 & 6.78 & $0.33^{* *}$ & $0.25^{* *}$ & $0.20 * *$ & & \\
\hline BSRS (T2) & 46.32 & 7.57 & $0.24^{* *}$ & $0.33^{* *}$ & $0.20^{* *}$ & $0.46^{* *}$ & \\
\hline BSRS (T3) & 45.24 & 7.95 & $0.20^{* *}$ & $0.23^{* *}$ & $0.36^{* *}$ & $0.37^{* *}$ & $0.44^{* *}$ \\
\hline
\end{tabular}

PMHS, Positive Mental Health Scale; BSRS, Brief Social Rhythm Scale; T1, baseline; T2, 1-year-follow-up; T3, 2-year-follow-up; ** p < 0.01. 


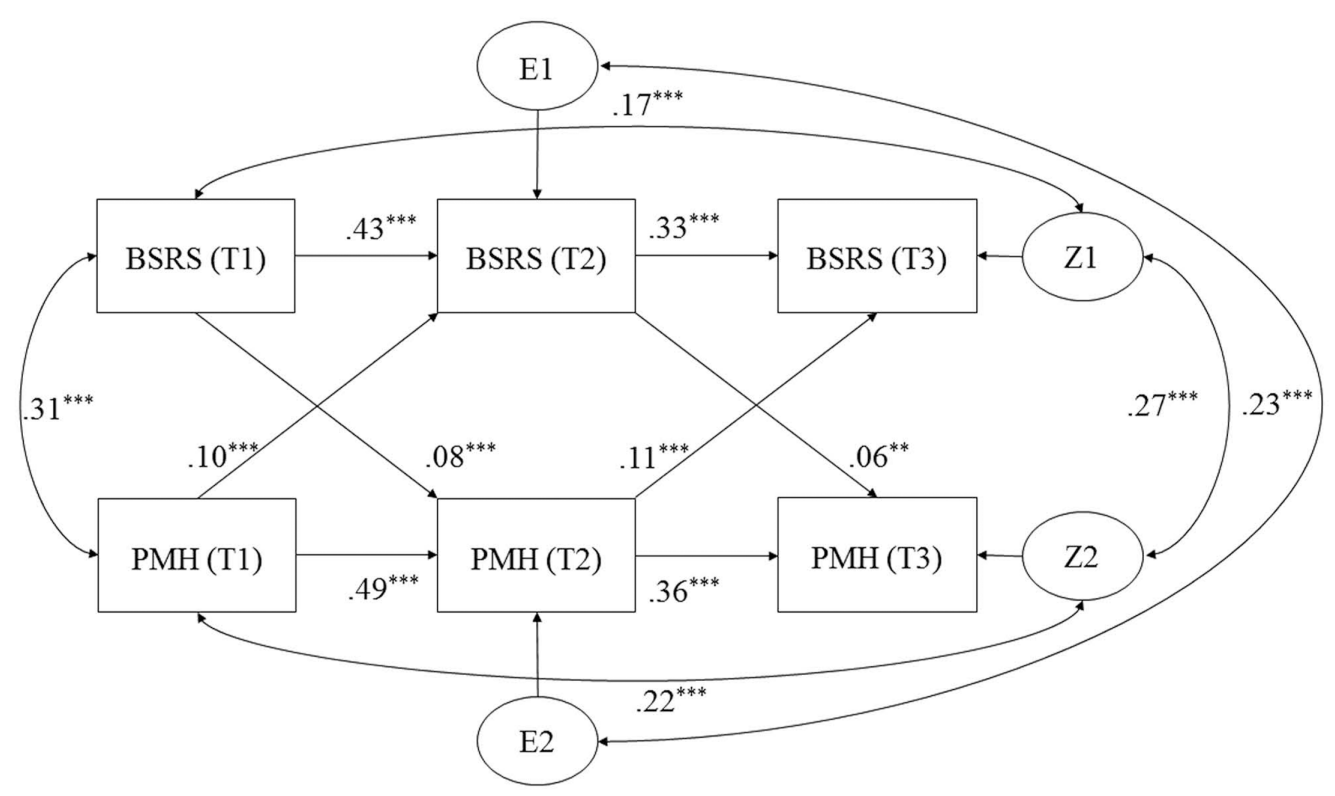

FIGURE 1 | Cross-lagged panel model for social rhythm measured by Brief Social Rhythm Scale (BSRS) and positive mental health measured by Positive Mental Health Scale (PMHS). T1, baseline; T2, 1-year-follow-up; T3, 2-year-follow-up; double-headed arrow, correlation; one-head arrow, regression. E1, E2, Z1, and Z2 represent the residual errors for BSRS (T2), PMHS (T2), BSRS (T3), and PMHS (T3), respectively. ${ }^{* *} p<0.01 ;{ }^{* * *} p<0.001$.

positive mental health, indicating that social rhythm regularity predicted the level of positive mental health a year later, and vice versa. These findings broaden the cross-sectional results in earlier studies and extend the understanding of the relationship of social rhythm and emotional well-being in younger adults.

The present study is innovative in several ways. First, as expected, social rhythm regularity was a strong predictor of positive mental health, suggesting that improving social rhythm may help to increase psychological well-being in college students. One explanation could be that enhanced rhythm regularity would directly promote one's mental well-being. For instance, several studies have pointed out that a regular daily rhythm would benefit sleep (Ohayon et al., 2001; Morgan, 2003), and in turn good quality sleep may increase the positive aspects of mental health, such as subjective well-being (Hamilton et al., 2007; Peach et al., 2016). An alternative but not incompatible explanation is that social rhythm regularity would decrease the level of mental symptoms or chances of onset of mental illness (Ehlers et al., 1988; Grandin et al., 2006; Shen et al., 2008; Bullock et al., 2011; Nusslock and Frank, 2012; Lieverse et al., 2013), promoting better mental health (Lamers et al., 2015). For example, it has been shown that social factors such as shift work and irregular working hours may desynchronize normal circadian rhythms (Costa, 1996), elevate stress response (Ulhoa et al., 2011), and lead to mental problems such as chronic fatigue, anxiety (Cole et al., 1990), and depressed mood (Driesen et al., 2010). Thus, a relatively regular schedule may lower the negative aspect of body and mental health, and lead to better positive mental health. Future research is warranted to clarify the potential mediators (e.g., changes in biological rhythm, increase in good sleep quality, or decrease in mental illness) of this bidirectional relationship.

Secondly, the reciprocal relationship displayed that positive mental health was also a consistent predictor of social rhythmicity. This result was consistent with some earlier findings, such as that positive affect and eudaimonic well-being are directly associated with better sleep quality and may buffer the impact of negative psychosocial factors (Steptoe et al., 2008), and that aging women with higher levels of eudaimonic well-being had longer duration of REM sleep compared to those showing lower levels of eudaimonic well-being (Ryff et al., 2004).

Furthermore, while the majority of previous studies used positive mental health only as an outcome measure indexing a desired state of mind (Steptoe et al., 2008), having positive mental health and emotions also proved to have many benefits, such as enhancing job performance in the following year (Cropanzano and Wright, 1999), protecting individuals against physical decline in old age (Ostir et al., 2000), and predicting longevity (Diener and Chan, 2011). However, only a few studies have considered positive mental health as predictive factor (Teismann et al., 2016; Lukat et al., 2017). Our empirical results add to this by pointing out that higher level of positive mental health helps to increase social rhythm regularity which was found to be associated with better mental state and less mental illness (Margraf et al., 2016).

In addition, the present study provided a picture of how psychological well-being and social rhythm fluctuate in college students across time. On the one hand, the results indicated that this population is indeed at risk of increasing irregularity in their daily rhythm as they proceed with their studies, which is in line with previous studies showing that younger and single 
populations were more irregular in their social rhythm compared to older people or to those living with a partner and children (van Tienoven et al., 2014). On the other hand, the current findings indicated that the psychological well-being in college students fluctuated over the years, and on a similar level in German, Russian, and United States samples reported in an earlier study (Margraf et al., 2016). These results also suggested that mental health of college students is not a fixed factor but can change over time (Xin et al., 2012), emphasizing the importance of additional longitudinal surveys regarding this topic.

There are some limitations to the current study. First, the sample included only college students, therefore, the results cannot be generalized to other adult populations without caution. Second, positive mental health consists of more than just emotional well-being (Baumeister et al., 2013), and "well-being" itself is composed of different aspects, such as social well-being or life-satisfaction. Consequently, when various components of positive mental health are considered, a differential pattern of the relationship between social rhythm and positive mental health might unfold (Lamers et al., 2012; Bieda et al., 2016). Future studies should address the various components of positive of mental health and well-being. Third, a large majority of the longitudinal sample was female, which could impact the representativeness of the results. However, the model comparisons demonstrated that the gender factor did not appear to have a significant impact on the final results.

\section{CONCLUSION}

In sum, the current study provides preliminary evidence of a mutually enhancing relationship between social rhythm and positive mental health with a 3 -year longitudinal design in a large college student sample. Further studies should take other components of positive mental health into account and consider

\section{REFERENCES}

Arbuckle, J. L. (2013). Amos, Version 22.0 [Software]. Chicago, IL: IBM Corp.

Baumeister, R. F., Vohs, K. D., Aaker, J. L., and Garbinsky, E. N. (2013). Some key differences between a happy life and a meaningful life. J. Posit. Psychol. 8, 505-516. doi: 10.1080/17439760.2013.830764

Bieda, A., Hirschfeld, G., Schönfeld, P., Brailovskaia, J., Zhang, X. C., and Margraf, J. (2016). Universal happiness? Cross-cultural measurement invariance of scales assessing positive mental health. Psychol. Assess. 29, 408-421. doi: 10.1037/pas0000353

Boland, E. M., Bender, R. E., Alloy, L. B., Conner, B. T., LaBelle, D. R., and Abramson, L. Y. (2012). Life events and social rhythms in bipolar spectrum disorders: an examination of social rhythm sensitivity. J. Affect. Disord. 139, 264-272. doi: 10.1016/j.jad.2012.01.038

Brown, L. F., Reynolds, C. F., Monk, T. H., Prigerson, H. G., Dew, M. A., Houck, P. R., et al. (1996). Social rhythm stability following late-life spousal bereavement: associations with depression and sleep impairment. Psychiatry Res. 62, 161-169. doi: 10.1016/0165-1781(96)02914-9

Bullock, B., Judd, F., and Murray, G. (2011). Social rhythms and vulnerability to bipolar disorder. J. Affect. Disord. 135, 384-388. doi: 10.1016/j.jad.2011. 06.006 a more comprehensive picture of this reciprocal relationship and its stability.

\section{AUTHOR CONTRIBUTIONS}

All authors agree to be accountable for the content of the work. DC designed the study and organized data collecting. MZ collected data, did the main statistical analyses, and draft part of the manuscript. ML did part of the statistical analyses and wrote and revised the manuscript. XZ organized data collecting and supervised statistical analyses. JM designed the study and the project, and reviewed the paper.

\section{FUNDING}

The study was facilitated by Alexander von Humboldt Professorship awarded to JM by the Alexander von HumboldtFoundation. The study is also supported by the National Natural Science Foundation of China (31600906) and Shanghai Pujiang Program (16PJC070) awarded to DC.

\section{ACKNOWLEDGMENTS}

We want to acknowledge Helen Copeland-Vollrath for proofreading the article, and the Open Access Publication Funds of the Ruhr-Universität Bochum for its support.

\section{SUPPLEMENTARY MATERIAL}

The Supplementary Material for this article can be found online at: http://journal.frontiersin.org/article/10.3389/fpsyg. 2017.01119/full\#supplementary-material

Cole, R. J., Loving, R. T., and Kripke, D. F. (1990). Psychiatric aspects of shiftwork. Occup. Med. 5, 301-314.

Costa, G. (1996). The impact of shift and night work on health. Appl. Ergon. 27, 9-16. doi: 10.1016/0003-6870(95)00047-X

Cropanzano, R., and Wright, T. A. (1999). A 5-year study of change in the relationship between well-being and job performance. Consult. Psychol. J. Pract. Res. 51, 252. doi: 10.1037/1061-4087.51.4.252

Diener, E., and Chan, M. Y. (2011). Happy people live longer: subjective well-being contributes to health and longevity. Appl. Psychol. Health Well Being 3, 1-43. doi: 10.1111/j.1758-0854.2010.01045.x

Driesen, K., Jansen, N. W., Kant, I., Mohren, D. C., and Van Amelsvoort, L. G. (2010). Depressed mood in the working population: associations with work schedules and working hours. Chronobiol. Int. 27, 1062-1079. doi: 10.3109/ 07420528.2010.489877

Ehlers, C. L., Frank, E., and Kupfer, D. J. (1988). Social zeitgebers and biological rhythms: a unified approach to understanding the etiology of depression. Arch. Gen. Psychiatry 45, 948-952. doi: 10.1001/archpsyc.1988.01800340076012

Frank, E., Kupfer, D. J., Thase, M. E., Mallinger, A. G., Swartz, H. A., Fagiolini, A. M., et al. (2005). Two-year outcomes for interpersonal and social rhythm therapy in individuals with bipolar I disorder. Arch. Gen. Psychiatry 62, 996-1004. doi: 10.1001/archpsyc.62.9.996 
Gorwood, P. (2012). Anxiety disorders and circadian rhythms. Medicographica 34, 289-294.

Grandin, L. D., Alloy, L. B., and Abramson, L. Y. (2006). The social zeitgeber theory, circadian rhythms, and mood disorders: review and evaluation. Clin. Psychol. Rev. 26, 679-694. doi: 10.1016/j.cpr.2006.07.001

Hamilton, N. A., Nelson, C. A., Stevens, N., and Kitzman, H. (2007). Sleep and psychological well-being. Soc. Indic. Res. 82, 147-163. doi: 10.1007/s11205-0069030-1

Harvey, A. G., Jones, C., and Schmidt, D. A. (2003). Sleep and posttraumatic stress disorder: a review. Clin. Psychol. Rev. 23, 377-407. doi: 10.1016/S0272-7358(03) 00032-1

Haynes, P. L., Kelly, M., Warner, L., Quan, S. F., Krakow, B., and Bootzin, R. R. (2016). Cognitive behavioral social rhythm group therapy for veterans with posttraumatic stress disorder, depression, and sleep disturbance: results from an open trial. J. Affect. Disord. 192, 234-243. doi: 10.1016/j.jad.2015.12.012

Hu, L. T., and Bentler, P. M. (1999). Cutoff criteria for fit indexes in covariance structure analysis: conventional criteria versus new alternatives. Struct. Equ. Model. Multidiscip. J. 6, 1-55. doi: 10.1080/10705519909540118

IBM Corp (2010). IBM SPSS Statistics for Windows, Version 19. 0 [Software]. Armonk, NY: IBM Corp.

Kendler, K. S., Myers, J. M., Maes, H. H., and Keyes, C. L. (2011). The relationship between the genetic and environmental influences on common internalizing psychiatric disorders and mental well-being. Behav. Genet. 41, 641-650. doi: 10.1007/s10519-011-9466-1

Kobayashi, I., Boarts, J. M., and Delahanty, D. L. (2007). Polysomnographically measured sleep abnormalities in PTSD: a meta-analytic review. Psychophysiology 44, 660-669. doi: 10.1111/j.1469-8986.2007.537.x

Kraemer, H. C., Kazdin, A. E., Offord, D. R., Kessler, R. C., Jensen, P. S., and Kupfer, D. J. (1997). Coming to terms with the terms of risk. Arch. Gen. Psychiatry 54, 337-343. doi: 10.1001/archpsyc.1997.01830160065009

Lamers, S. M., Westerhof, G. J., Glas, C. A., and Bohlmeijer, E. T. (2015). The bidirectional relation between positive mental health and psychopathology in a longitudinal representative panel study. J. Posit. Psychol. 10, 553-560. doi: $10.1080 / 17439760.2015 .1015156$

Lamers, S. M., Westerhof, G. J., Kovács, V., and Bohlmeijer, E. T. (2012). Differential relationships in the association of the Big Five personality traits with positive mental health and psychopathology. J. Res. Pers. 46, 517-524. doi: 10.1016/j.jrp.2012.05.012

Lieverse, R., de Vries, R., Hoogendoorn, A. W., Smit, J. H., and Hoogendijk, W. J. (2013). Social support and social rhythm regularity in elderly patients with major depressive disorder. Am. J. Geriatr. Psychiatry 21, 1144-1153. doi: 10.1016/j.jagp.2013.01.052

Lukat, J., Becker, E. S., Lavallee, K. L., Veld, W. M., and Margraf, J. (2017). Predictors of incidence, remission and relapse of Axis I mental disorders in young women: a transdiagnostic approach. Clin. Psychol. Psychother. 24, 322-331. doi: 10.1002/cpp.2026

Lukat, J., Margraf, J., Lutz, R., van der Veld, W. M., and Becker, E. S. (2016). Psychometric properties of the positive mental health scale (PMH-scale). BMC Psychol. 4:8. doi: 10.1186/s40359-016-0111-x

Margraf, J., Lavallee, K., Zhang, X. C., and Schneider, S. (2016). Social rhythm and mental health: a cross-cultural comparison. PLOS ONE 11:e0150312. doi: 10.1371/journal.pone.0150312

Meyer, T. D., and Maier, S. (2006). Is there evidence for social rhythm instability in people at risk for affective disorders? Psychiatry Res. 141, 103-114. doi: 10.1016/j.psychres.2005.07.023

Morgan, K. (2003). Daytime activity and risk factors for late-life insomnia. J. Sleep Res. 12, 231-238. doi: 10.1046/j.1365-2869.2003.00355.x

Nusslock, R., and Frank, E. (2012). "Interpersonal social rhythm therapy (IPSRT) for bipolar disorder," in Casebook of Interpersonal Psychotherapy, eds J. C. Markowitz and M. M. Weissman (New York, NY: Oxford University Press), 103-121. doi: 10.1093/med:psych/9780199746903.003.0007

Ohayon, M. M., Zulley, J., Guilleminault, C., Smirne, S., and Priest, R. G. (2001). How age and daytime activities are related to insomnia in the general population: consequences for older people. J. Am. Geriatr. Soc. 49, 360-366. doi: 10.1046/j.1532-5415.2001.49077.x

Ostir, G. V., Markides, K. S., Black, S. A., and Goodwin, J. S. (2000). Emotional well-being predicts subsequent functional independence and survival. J. Am. Geriatr. Soc. 48, 473-478. doi: 10.1111/j.1532-5415.2000.tb0 4991.x

Peach, H., Gaultney, J. F., and Gray, D. D. (2016). Sleep hygiene and sleep quality as predictors of positive and negative dimensions of mental health in college students. Cogent Psychol. 3:1168768. doi: 10.1080/23311908.2016.1168768

Penedo, F. J., and Dahn, J. R. (2005). Exercise and well-being: a review of mental and physical health benefits associated with physical activity. Curr. Opin. Psychiatry 18, 189-193. doi: 10.1097/00001504-200503000-00013

Ryff, C. D., Singer, B. H., and Love, G. D. (2004). Positive health: connecting wellbeing with biology. Philos. Trans. R. Soc. Lond. B Biol. Sci. 359, 1383-1394. doi: $10.1098 /$ rstb.2004.1521

Shen, G. H., Alloy, L. B., Abramson, L. Y., and Sylvia, L. G. (2008). Social rhythm regularity and the onset of affective episodes in bipolar spectrum individuals. Bipolar Disord. 10, 520-529. doi: 10.1111/j.1399-5618.2008.00583.x

Steptoe, A., O’Donnell, K., Marmot, M., and Wardle, J. (2008). Positive affect, psychological well-being, and good sleep. J. Psychosom. Res. 64, 409-415. doi: 10.1016/j.jpsychores.2007.11.008

Sylvia, L. G., Alloy, L. B., Hafner, J. A., Gauger, M. C., Verdon, K., and Abramson, L. Y. (2009). Life events and social rhythms in bipolar spectrum disorders: a prospective study. Behav. Ther. 40, 131-141. doi: 10.1016/j.beth.2008.04.003

Teismann, T., Forkmann, T., Glaesmer, H., Egeri, L., and Margraf, J. (2016). Remission of suicidal thoughts: findings from a longitudinal epidemiological study. J. Affect. Disord. 190, 723-725. doi: 10.1016/j.jad.2015.09.066

Thase, M. E., Jindal, R., and Howland, R. H. (2002). "Biological aspects of depression," in Handbook of Depression, eds I. H. Gotlib and C. L. Hammen (New York, NY: Guildford Press), 192-218.

Ulhoa, M. A., Marqueze, E. C., Kantermann, T., Skene, D., and Moreno, C. (2011). When does stress end? Evidence of a prolonged stress reaction in shiftworking truck drivers. Chronobiol. Int. 28, 810-818. doi: 10.3109/07420528.2011.613136

van Tienoven, T. P., Minnen, J., Daniels, S., Weenas, D., Raaijmakers, A., and Glorieux, I. (2014). Calculating the social rhythm metric (SRM) and examining its use in interpersonal social rhythm therapy (IPSRT) in a healthy population study. Behav. Sci. 4, 265-277. doi: 10.3390/bs4030265

Velten, J., Lavallee, K. L., Scholten, S., Meyer, A. H., Zhang, X. C., Schneider, S., et al. (2014). Lifestyle choices and mental health: a representative population survey. BMC Psychol. 2:58. doi: 10.1186/s40359-014-0055-y

Weich, S., Brugha, T., King, M., McManus, S., Bebbington, P., Jenkins, R., et al. (2011). Mental well-being and mental illness: findings from the Adult Psychiatric Morbidity Survey for England 2007. Br. J. Psychiatry 199, 23-28. doi: 10.1192/bjp.bp.111.091496

Westerhof, G. J., and Keyes, C. L. (2010). Mental illness and mental health: the two continua model across the lifespan. J. Adult Dev. 17, 110-119. doi: 10.1007/ s10804-009-9082-y

World Health Organization (2004). Promoting Mental Health: Concepts, Emerging Evidence, Practice: Summary Report. Geneva: World Health Organization.

Xin, Z. Q., Zhang, M., and He, L. (2012). Changes in college students' mental health: a cross-temporal meta-analysis. Acta Psychol. Sin. 44, 664-679. doi: 10.3724/SP.J.1041.2012.00664

Conflict of Interest Statement: The authors declare that the research was conducted in the absence of any commercial or financial relationships that could be construed as a potential conflict of interest.

Copyright $\odot 2017$ Cai, Zhu, Lin, Zhang and Margraf. This is an open-access article distributed under the terms of the Creative Commons Attribution License (CC BY). The use, distribution or reproduction in other forums is permitted, provided the original author(s) or licensor are credited and that the original publication in this journal is cited, in accordance with accepted academic practice. No use, distribution or reproduction is permitted which does not comply with these terms. 